IAU Colloquium 190 on Magnetic Cataclysmic Variables

ASP Conference Series, Vol. 315, 2004

Sonja Vrielmann 83 Mark Cropper, eds.

\title{
Dynamo-Induced Mass-Transfer Variations in MCVs
}

\author{
F.V. Hessman \\ Universitäts-Sternwarte, Geismarlandstr. 11, 37083 Göttingen, \\ Germany
}

\begin{abstract}
The fully convective secondary stars in short-period CVs are rapidly rotating and thus should have a characteristic global magnetic field produced by an $\alpha^{2}$-Dynamo. This field is expected to be dipole-like, with an axis lying in the orbital plane, and to rotate slowly through the mass of the star on magnetic diffusion timescales of years to decades. When one of the poles of this field is near the inner Lagrange point, the reduction in the sound speed, scale-height and possibly the density in the giant "starspot" will modulate the mass-transfer to the primary. Given the extremely high Coriolis numbers (low Rosby numbers) of CV secondaries, this is a generic mechanism which is capable of explaining the extended high- and low-states in the mean light curves of shorter period MCVs and perhaps the lightcurves of non-magnetic CVs (where a disk may make it difficult to see this effect). The diffusion timescale and hence the period of rotation of the field within the secondary is of order the synchronization timescale, so shorter period polar's are unlikely to be in rotational equilibrium.
\end{abstract}

\section{Mass-Transfer Variations and their Cause}

All cataclysmic variables are (almost by definition) photometrically variable. However, polars have the unique characteristic that the material in the accretion stream isn't halted by an accretion disc before impacting on the surface of the primary, so the photometric variations are fairly direct measures of the nearinstantaneous mass-transfer rate (see Hessman, Gänsicke \& Mattei 2000 for a prescription for a visual bolometric correction).

Figure 1 shows the estimated mass-transfer rate in AM Her during the last $\sim 9000$ days (courtesy of J. Mattei \& B. Gänsicke). Unlike normal dwarf novae, polar have long periods of several years during which the mean mass-transfer rate is either very low or very high, punctuated by rapid and much more shortlived excursions with timescales of days, weeks, or months. While the long-term changes could, in principle, be due to mean radius changes produced by global stellar activity cycles (e.g. Warner 1988), the extremely short timescales of only days for some of the transitions suggests that the ultimate cause of the variations is the passage or evolution of starspots at the inner Lagrangian point $\mathrm{L}_{1}$ (e.g. Livio \& Pringle 1994).

It is easy to see how starspots could modulate the mass-transfer rate: the rate depends upon the sound speed and density of material in the atmosphere of 


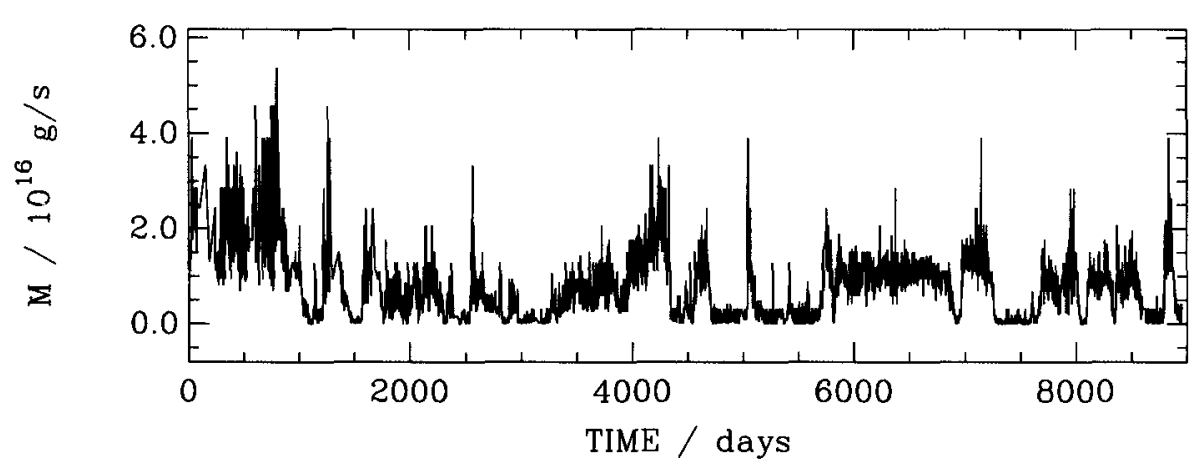

Figure 1. Daily mass-transfer rate in AMHer, estimated from AAVSO lightcurves, courtesy of J. Mattei \& B. Gänsicke.

the secondary at $\mathrm{L}_{1}$. Dark starspots by definition have lower temperatures and sound speeds than the surrounding atmophere and the decrease in the atmospheric scale height probably reduces the density as well. Unfortunately, solar physicists don't understand the detailed structure of even sunspots much less starspots well enough to predict the exact effect in CVs so it is impossible to invert the mass-transfer lightcurve into a measure of, e.g., the instantaneous magnetic fields. For instance, the well-known "Wilson-Depression" in sunspots is an optical depth effect which may or may not have anything to do with the mass-density. We do know that spot groups in rapidly rotating stars can be very large, particularly when they are found at the rotational pole (see A.N. 323, 2002 for a recent compendium), very similar to the high mean spottedness of around $75 \%$ at the $\mathrm{L}_{1}$-point estimated for AM Her (Hessman, Gänsicke \& Mattei 2000). The question is, what form does the spottedness take in CV secondaries? Are the spots distributed randomly or in some global overall pattern? If there is a pattern, is it spatial or temporal? Looking at the mass-transfer curve of AM Her (Fig. 1), one can imagine that there is a rough long-term variation with a period of about 2000 days ( $5 \frac{1}{2}$ years). In the starspot model, this is an indication that the spots are not distributed totally randomly, but are grouped into spatial or temporal structures.

AMHer isn't alone in showing long-term variations in its accretion rate. Ak, Ozkan \& Mattei (2001) have studied the light curves of a number of nonmagnetic CVs and have found periodic variations in, e.g., the quiescent light level or the frequency of dwarf nova eruptions. The characteristic timescales range from about a half to several decades and show no obvious correlation with any orbital or stellar properties. Again, a long-term smooth variation in $\dot{M}$ could be caused by, e.g., a third star around the binary, but there's no reason why these variations couldn't be identical to the much more eratic variation in polars. 


\section{Dynamos in CV Secondaries}

The secondaries in CVs are rapidly rotating, partially- or fully convective latetype stars, so we expect them to have dynamos which create the starspots needed to explain the mass-transfer variations. The equation governing a simple meanfield kinematic dynamo is

$$
\frac{\partial \boldsymbol{B}}{\partial t}=\nabla \times(\mathrm{v} \times \boldsymbol{B}+\boldsymbol{\alpha} \boldsymbol{B})+\eta_{T} \nabla^{2} \boldsymbol{B}
$$

where $\eta_{T}$ is the magnetic eddy diffusivity, and $\boldsymbol{\alpha}$ is a tensor describing how turbulent or meridinal motions can convert poloidal magnetic fields into helical fields and vice versa. The first term on the right hand side shows that gradients in the magnetohydrodynamic velocity field are needed: these can be produced either on a large scale via differential rotation (the so-called " $\Omega$-effect"), largescale meridinal motions, or by the turbulent velocity field. The timescale for global magnetic field variations can be estimated using the mean magnetic eddy diffusivity of the sun

$$
t_{\text {diff }} \approx \frac{\Delta R R}{<\eta_{T}>} \approx 15 y r\left(\frac{\Delta R R}{0.1 R_{\odot}^{2}}\right)\left(\frac{<\eta_{T}>}{10^{12} c m^{2} s^{-1}}\right)^{-1}
$$

where $\Delta R$ is the size of the relevant convective region ( $=R$ in fully convective stars).. The dynamos in partially convective stars like our sun are of the " $\alpha-\Omega$ "type, where the differential rotation of the star $(\Omega)$ shears and thereby enhances the field $(\alpha)$ in the partially convective layer between the radiative core and the convective envelope. Fully convective stars, on the other hand, are expected to rotate nearly uniformly, so the dynamo effect is produced by the non-uniform convective and meridinal velocity field: the so-called $\alpha^{2}$-dynamo.

Secondary stars in CVs above the period gap (like AM Her) may have radiative cores, but they are unlikely to have much differential rotation, so the main dynamo effect for shorter period CV secondaries should be of the $\alpha^{2}$ type. Numerical simulations of $\alpha^{2}$-dynamos (e.g. Moss \& Brandenburg 1995) in fully convective late dwarfs (e.g. Küker \& Rüdiger 1999; Kitchatinov, Jardine \& Collier Cameron 2001) show that such rapidly rotating stars will have quasi-dipolar fields with axes perpendicular to the rotation axis (i.e. in the orbital plane of CVs: see Moss \& Brandenburg for visualizations of the fields) and that the fields slowly rotate relative to the star on a magnetic diffusion timescale (Eqn. 2). The measure of what is "rapidly rotating" is that the rotation period should be shorter than or of order the convective turnover time, i.e. the so-called Coriolis number

$$
\Omega_{*} \equiv \frac{4 \pi}{R o} \equiv \frac{4 \pi t_{\text {conv }}}{P_{\text {rot }}}>\text { about } 3
$$

where $R o$ is the Rosby number, the ratio of the rotation period $P_{\text {rot }}$ to the convective turnover time, $t_{c o n v}$. The convective turnover times in low-mass stars $\left(M \lesssim 0.3 M_{\odot}\right)$ derived from detailed models (Baraffe, private communication) is roughly

$$
t_{\text {conv }} \approx 460^{d}\left(\frac{M}{0.1 M_{\odot}}\right)^{-1.3}
$$


and the rotation period of CV secondaries is of the order of hours, so the typical Coriolis numbers are of order $10^{4} \gg 3$. Thus, the magnetic field pattern in all short-period CV secondaries should be that predicted by $\alpha^{2}$-models: dipolar, axis in the orbital plane, and rotating through the star on a magnetic diffusion timescale of years to decades.

Since there is no reason to believe that $\eta_{T}$ is a universal constant, we can use Eqn. 2 and the observed timescale for variations (from Ak, Ozkan \& Mattei 2001) for the well-measured low-mass secondaries in OY Car and Z Cha $\left(R_{2}=0.13\right.$ and $0.17 M_{\odot}$, and $P_{\text {cycle }}=6.3$ and 14.6 years, respectively) to obtain a magnetic eddy diffusivity for low-mass stars of about $210^{11} \mathrm{~cm}^{2} \mathrm{~s}^{-1}$ : the timescale for magnetic field changes in low-mass stars can be about the same as for higher mass stars with much larger radii if the eddy diffusivity is lower. Unfortunately, this value of $\eta_{T}$ isn't too reliable, since the values of $P_{\text {cycle }}$ found by Ak, Ozkan \& Mattei show much spread for seemingly similar stars. Nevertheless, the expected dynamo timescales are easily in the range of the observed mass-transfer cycle timescales.

\section{Consequences for MCVs}

With the conclusion that all secondaries in shorter-period CVs should have dipole-ish fields with axes in the orbital plane which rotate in the co-rotating frame of the binary on timescales of years to decades, we can explain the quasisinusoidal long-term mass-transfer variations seen in polars and non-magnetic CVs as being the effect of the poles rotating slowing in and out of $\mathrm{L}_{1}$. The short-term variations are also easily explained as being due to the sub-structure within the globally dipole-ish field - either individual starspots or bright photospheric spots within dense groups of starspots, depending upon the direction of the change. Proof of this hypothesis may eventually come from Doppler imaging, where one predicts that the secondaries should have large spots at $L_{1}$ and on the opposite side of the secondary during low states.

This model also has profound consequences for the rotational states of polars. The possible equilibrium configurations of the magnetic fields of the two stars in polars have been studied by Campbell (1989) and King, Frank \& Whitehurst (1990). Ignoring the accretion torque, one can show that the dipole moments $\boldsymbol{\mu}_{1}, \boldsymbol{\mu}_{2}$, and the line separating the two stars must be coplanar. In our case, the secondary's $\alpha^{2}$-dynamo automatically defines that plane as the orbital plane. Since $\boldsymbol{\mu}_{2}$ is effectively held "fixed" by the dynamo on the timescale of months and the secondary's large moment of inertia - the primary is a crude magnetic compass following the field of the secondary - the only equilibrium configurations are $\boldsymbol{\mu}_{i}$ 's either (anti-)aligned or perpendicular with the separation vector (while remaining in the orbital plane). This configuration only occurs for a fraction of the dynamo period. The inclusion of the accretion torque changes the stable configurations only slightly, so the second major conclusion is that the two magnetic stars cannot be in rotational equilibrium. Even worse, it is easy to show that the timescale for synchronization of the two magnetic stars in polars in the case of maximum synchronization torque is of the order $t_{\text {diff }}$ (Campbell 1983), i.e. the timescale of the dynamo field rotation, so the configuration shouldn't have enough time to be even in quasi-equilibrium. 
The final conclusion must be that the primary's magnetic field cannot be oriented in a quasi-stationary direction in the rotating frame of the binary. This is in stark contrast to the canonical view of polars, where only a few are known to show obvious signs of non-synchronous rotation. Observations suggest that the final impact point of the accretion stream material clusters about a particular direction (about $20-30^{\circ}$ from the line of centers and from the orbital axis). Despite the fact that the primary is completely out of rotational equilibrium, the evolution of the alignments is apparently such that no change in the longitude and latitude for the accretion exists. In order to understand this behavior, it is necessary to compute the full torques on both stars, including the modulation of the accretion torque by the dynamo field (less accretion torque while the secondary's magnetic poles are near the $\mathrm{L}_{1}$-point) and the fact that the magnetic torques also transfer angular momentum to/from the orbit (King, Frank \& Whitehurst 1990). A detailed study of the resulting dynamics of the stellar and orbital rotation is now underway (Hessman, in preparation).

\section{Conclusions}

Current $\alpha^{2}$-dynamo models make a firm prediction of the behavior of the global magnetic fields in all shorter-period CV secondaries (i.e. those with no or small radiative cores). The major dipole component rotates slowly through the star in the plane of the orbit and so should cause mass-transfer variations on the timescales of many years to decades, as is observed. In polars, the interaction of the dynamo field with the primary's field will cause the primary to be out of rotational equilibrium. Simulations are being performed to see what observational consequences this interaction may have.

\section{References}

Ak, T., Ozkan, M.T., \& Mattei, J.A. 2001, A\&A, 369, 882

Campbell, C.G. 1983, MNRAS, 205, 1031

Campbell, C.G. 1989 , MNRAS, 236, 475

Cropper, M. 1990, Space Sci.Rev., 54, 195

Hessman, F.V., Gänsicke, B., \& Mattei, J.A. 2000, A\&A, 361, 952

King, A.R., Frank, J., \& Whitehurst, R. 1990, MNRAS, 244, 731

Kitchatinov, L.L., Jardine, M., \& Collier Cameron, A. 2001, A\&A, 374, 250

Küker, M. \& Rüdiger, G. 1999, A\&A, 346, 922

Livio, M. \& Pringle, J.E. 1994, ApJ, 427, 956

Moss, D. \& Brandenburg, A. 1995, Geophys. Ap. Fluid Dyn., 80, 229

Warner, B. 1996, Nature, 336, 129 